\begin{tabular}{|c|c|}
\hline \multirow{3}{*}{ 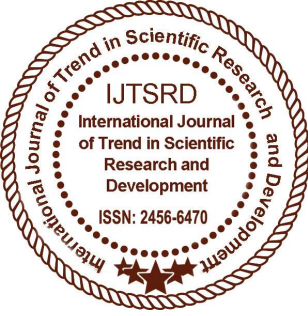 } & $\begin{array}{l}\text { International Journal of Trend in Scientific } \\
\text { Research and Development (IJTSRD) }\end{array}$ \\
\hline & International Open Access Journal \\
\hline & ISSN No: 2456 - 6470 | www.ijtsrd.com | Volume - 2 | Issue - 4 \\
\hline
\end{tabular}

\title{
Accessing the Internet Through Light Using LiFi
}

\author{
Ms. Poonam Konde, Mr. Prashant Shimpi \\ Bharati Vidyapeeth's Institute of Management and Information Technology, \\ CBD Belapur, Navi Mumbai, India
}

\begin{abstract}
LiFi is a short form for Light Fidelity. This concept came in the picture during 2011 in a TED talk by Harald Hass, who is a German physicist. Because of him we came to know the concept of accessing the internet through light. Wi-Fi uses radio frequency and LIFI uses visible light communication (VLC). [1] The Data transmission in $\mathrm{LiFi}$ is faster than WiFi. LiFi uses light emitting diodes for transmission of data. Information transmission through VLC is much more fast so the human eye is cannot see it.
\end{abstract}

Keywords: LiFi, LED, Visible Light

\section{INTRODUCTION}

In early days we used to access the internet through Dial-Up connection, now internet has evolved a lot. We moved from Dial-Up connection to Broadband and from Broadband we moved to Wi-Fi.

Right now Wi-Fi is taking $50 \%$ of internet traffic data and these numbers are increasing because we are moving to Internet of Things now. There has been a gradual increase of WiFi usage. It is used in Railway stations, Hotels, Colleges, Cafes, Airports, Buses and Homes. In upcoming future each device will be connected to the internet.

Can WiFi handle the task to bring internet to every place in future? Probably not, because WiFi is facing certain problems. Wifi uses radio frequency to transmit wireless data. The current issue with the WiFi is that we have limited spectrum available with us and the spectrum is getting filled up now.[3] Another issue is licensing a new spectrum is a huge task. Throughout the globe it is very difficult to licensing a new spectrum and also having limited bandwidth available with WiFi. Another issue of WiFi is security. We cannot use Wifi in aircraft, healthcare inside ICU, underwater.

We can solve these problems with light Sources. Use light to transmit data instead of WiFi. So the same light source which gives you illumination can give you data transmission also. This is the technology LiFi. We use visible part of light spectrum to send data instead of radio frequency. Instead of using modems, LiFi uses LED bulbs with transceiver. LiFi has protocol-802.11 a/c. In Paris LiFi world's first office was opened.

It works same as TV REMOTE. In our TV REMOTE we have infrared blaster (or IR blaster) it has LED light due to which signal is sent to your TV. Same as in LIFI we use light because light has fastest speed 299,792,458 metres per second. In LiFi collection of led light in circuit which has drivers due to which more data is sent through LED. So you can use data. You need to have LiFi light to use LiFi technology. It is not harmful to your body because there are no radiations.

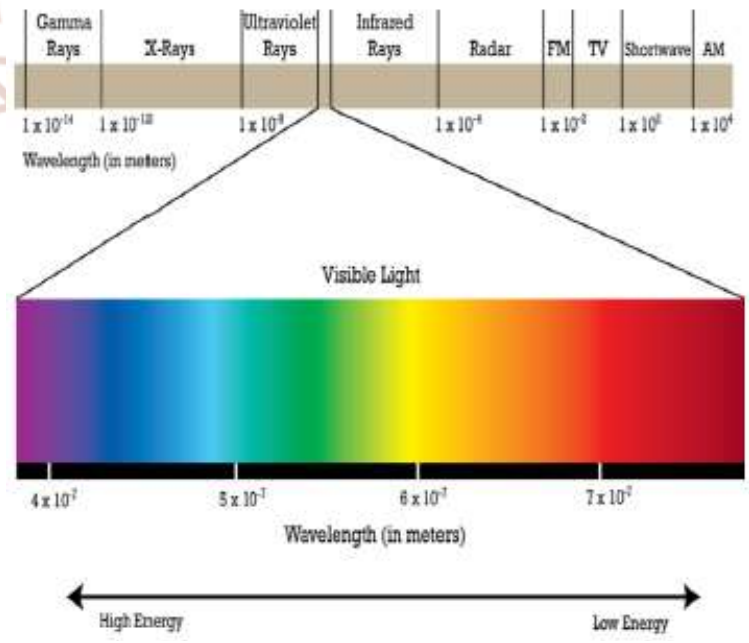

Fig: Electromagnetic spectrum 


\section{LITERATURE SURVEY}

1. LiFi has plus points like:

$>$ It has 10 times faster speed than Wi-Fi.

$>$ It is highly secure because the light which is present inside a room will not go out if the door is closed. So the data will remain in the room only.

$>$ We can use LiFi in aircraft to transmit the information.

$>$ It is future of $5 \mathrm{G}$ and $6 \mathrm{G}$ network.

\section{Education and Research network (ERNET):}

$>$ It is a scientific autonomous society where the testing of lifi was successful.

$>$ The use of Lifi can be used in the smart cities where the lifi can be used using connected LED bulbs.

$>$ Within a one-km radius the test was conducted at IIT-Madras for testing of a 10 Giga byte per second internet speed using light.

$>$ In rural areas where electricity is present in those areas through LiFi we can provide internet.[2]

\section{Philips VLC setup -}

$>$ For shoppers, Shop has Philips LED LIGHT. They have to download app in which LED light work with app. That will detect what product you buy and what is the discount on it.

> LiFi works brilliant in Dim Light also because the sensor in your phone/laptop can easily detect that light and catch data.

4. Recent research at oxford university a professor find out that the speed we can increase up to 224 Gigabyte per sec, in which we can download 18 high definition movies in one second.

5. Apple is looking forward to implement lifi capability in future version of iphone.

6. Now a company named pureLiFi Ltd. has invented a LiFi dongal.[4]

\section{HOW IT WORKS}

1. It has basically two parts one is a transmitter and other one is a receiver. The Transmitter is an LED lamp and the LED lamp has a property that switches very quickly. Basically all data is in binary format which is 0 and 1 which is understood by the computer. We bring the data to the light source and convert the data in 1 and 0 then the blub blinks. The blinking of light is at pretty fast rate so the LED which is blinking 1 and 0 continuously and sending information to the user. On the receiver's end there is a photo detector which can understand that light pattern and converts the data.

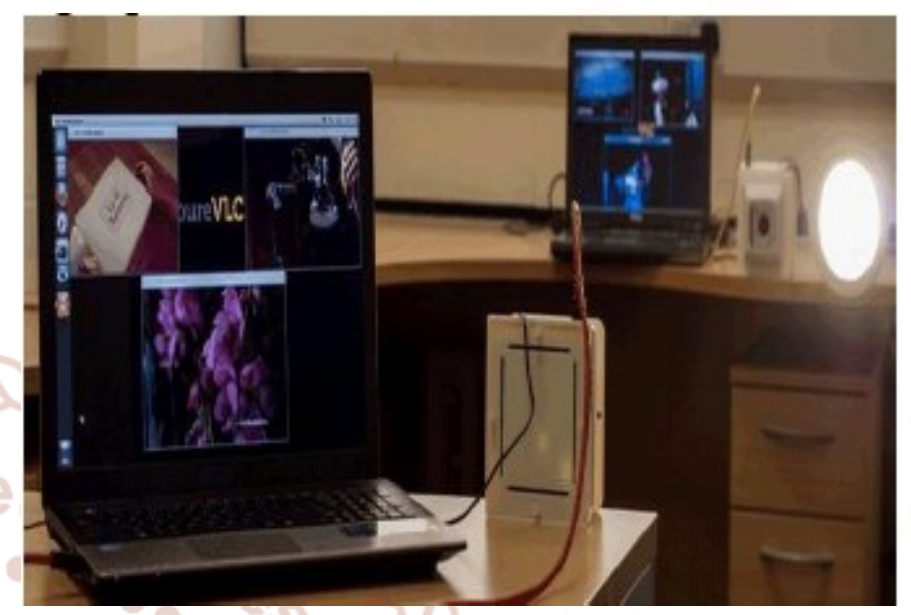

Fig: Demonstration of Lifi.

2. In above diagram there is a LED lamp, server. Server has video stored in it. The server is connected to lifi router and lifi router is connected to LED lamp which provides light and deliver access to the server. On the other end a laptop is connected with a lifi dongle which has blue element who is the receiver.

\section{ADVANTAGES}

\section{Connectivity:}

In Conference halls, classrooms, labs we can access internet everywhere with use of Light.

\section{Internet of Everything}

Since smart phone are coming up, everything is going to be connected to the internet so in that case we need a communication medium which can bring data from these devices to the internet and that could be the light. In these areas we use LiFi.[4]

\section{Telecom Sector}

In telecom sector we face problems for creating better internet infrastructure. We still don't have good internet in our country and its takes lot of energy from telecom sites to setup internet infrastructure. Means Telecom Company has to run optical cables under the street and poles. 
It creates lots of infrastructure issues. But with LiFi we already have light bulbs and we can utilize that. In future we can access internet through street bulbs.

\section{Aviation Industry}

In Aircraft we can watch movie, video through light so it can replace lots of cables in aircraft.[3] It provides security inside aircraft.

\section{Intelligence Transportation System}

In future our vehicles are going to be connected to each other. They can pass information about speed, traffic to other vehicles. We can utilize lights from cars or Street lamps.

\section{Indoor Navigation}

In smart retail store we can easily find the stuff. With the use of retail light we can create local map. [3]

\section{Smart Cities:}

Within smart cities we can create better infrastructure for $\mathrm{LiFi}$. It can replace in future the optical cable communication. Currently we have optical infrastructure in these area we could use Lifi.

\section{Stadium:}

In stadium there is a large area in which more crowds are present. In that area lots of lights are Available that light can be useful for internet access. [4]

\section{Petro-Chemical Plants:}

In Petro-chemical plants, Oil platform we cannot be able to use internet because there is chance of exposure of light due to radio frequency.

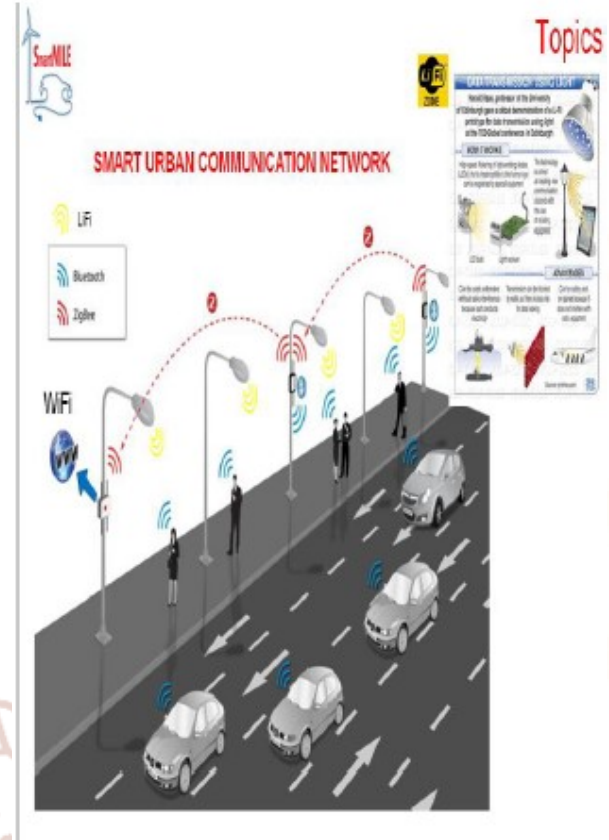

\section{CONCLUSION}

By this we conclude that with the existing infrastructure of available lights we can form Inter Light Net. If LiFi comes into the picture in future it would overcome all limitations of WiFi. The use of $\mathrm{LiFi}$ can replace radio based wireless technologies. $\mathrm{LiFi}$ is free and provides a secure and safe internet facility. This technology helps to solve the problem of short radio frequency bandwidth in hospitals and aircrafts. New Internet will be available which will be based on pure light sources.

\section{REFERENCES}

1. https://en.wikipedia.org/wiki/

2. http://trak.in/tags/business/2018/01/30/m inistrywants-lifi-india-tests-10-gbps-light-basedwireless-internet-iit-madras/

3. https://www.techworld.com

4. https://purelifi.com/technology/ 